\title{
Sexualidade e qualidade de vida em homens com dificuldades sexuais
}

\author{
Maria Cristina Romualdo Galati - Universidade Federal de São Paulo, São Paulo, Brasil \\ Enaury de Oliveira Alves Jr. - Universidade Federal de São Paulo, São Paulo, Brasil \\ Ana Claudia Crotti Delmaschio - Universidade Federal de São Paulo, São Paulo, Brasil \\ Ana Lucia de Moraes Horta - Universidade Federal de São Paulo, São Paulo, Brasil
}

\begin{abstract}
Resumo
O presente estudo teve por objetivo investigar a sexualidade e a qualidade de vida de homens com dificuldades sexuais. Foram selecionados dez homens que procuraram o serviço de sexualidade do CIESEX/UNIFESP. A triagem foi realizada através de uma entrevista estruturada contendo dados sociodemográficos e informações sobre as queixas apresentadas. Optou-se pela utilização dos questionários The Golombok-Rust Inventory of Sexual Satisfaction (GRISS) e Short Form Health Survey 36-item (SF-36), preenchidos pelos próprios entrevistados. Os resultados apontaram que homens com dificuldades sexuais apresentam uma menor qualidade em seu funcionamento sexual, bem como uma diminuição dos aspectos emocionais na qualidade de vida. Tal quadro revela um campo a ser explorado, demonstrando a necessidade de estudos que investiguem a correlação entre as dificuldades sexuais e qualidade de vida.

Palavras-chave: Disfunções sexuais; Qualidade de vida; Sexualidade.
\end{abstract}

\section{Sexuality and quality of life in men with sexual difficulties}

\begin{abstract}
The present study aimed to investigate sexuality and quality of life of men with sexual difficulties. We selectedten men who contacted the department ofsexuality CIESEX/UNIFESP. The screening was performed using a structured interview containing socio-demographic data and information on their complaints. We have chosentouse of questionnairesThe Golombok-Rust Inventory of Sexual Satisfaction(GRISS) and Short Form36-item Health Survey (SF-36), completed by the respondents. The results showed that men withs exual difficulties have lower performance in their sexual functioning, as well as a reduction in emotional quality of life. This table shows a field to be explored, demonstrating the need for studies to investigate the correlation between sexual difficulties and quality of life. Keywords: Sexual dysfunctions; Quality of life; Sexuality.
\end{abstract}

\section{Sexualidad y calidad de vida en hombres con dificultades sexuales}

\section{Resumen}

Este estudio tuvo como objetivo investigar la sexualidad y la calidad de vida de hombres con problemas sexuales. Fueron seleccionados diez hombres que buscaron el servicio de sexualidad del CIESEX/UNIFESP. La selección se realizó mediante una entrevista estructurada con datos sociodemográficos e informaciones sobre las quejas presentadas. Se optó por utilizar los cuestionarios The Golombok-Rust Inventory of Sexual Satisfaction (GRISS) y Short Form Health Survey 36-item (SF-36), respondidos por los propios participantes. Los resultados mostraron que los hombres con dificultades sexuales presentaron una menor calidad en su funcionamiento sexual, así como una reducción de los aspectos emocionales en la calidad de vida. Ese hecho muestra un campo a explorar, lo que demuestra la necesidad de realizar estudios para investigar la correlación entre las dificultades sexuales y la calidad de vida.

Palabras-clave: Disfunciones sexuales; Calidad de vida; Sexualidad.

\section{Introdução}

A sexualidade é um componente inerente à vida de todos os seres humanos, desenvolvendo-se em um processo contínuo, que se inicia antes mesmo do nascimento e só se encerra com a morte. A estruturação da sexualidade se dá sobre os aspectos biopsicossociais de cada indivíduo, abrangendo seu potencial biológico, suas emoções, sentimentos, crenças e concepções desenvolvidas, ampliadas e modificadas durante todo o processo de socialização. É também uma parte intercomunicante de um indivíduo consigo mesmo e com aqueles com os quais se relaciona ao longo de sua vida, influenciando a sua maneira de ser e de se posicionar no mundo que o cerca. Desde que o homem existe, há a preocupação em se compreender essa dimensão do ser humano, seja em sua vivência saudável, seja na patológica, normal ou desviante (Gherpelli, 1995).

Um dos modelos utilizados para a compreensão do funcionamento sexual é o ciclo da resposta sexual humana, desenvolvido por Masters e Johnson ao final da década de 60. Tal ciclo é caracterizado como uma sucessão de transformações físicas, cujo objetivo é preparar os corpos de dois parceiros para o ato sexual. Dividem-no em quatro fases sucessivas: excitação, platô, orgasmo e resolução (Masters \& Johnson, 1984). 
Posteriormente, Kaplan (1977) acrescenta o desejo sexual como o estágio inicial, desconsiderando a fase de platô.

Desejo, excitação, orgasmo e resolução são as etapas do ciclo sexual adotadas pelo DSM-IV (1995) para classificação dos transtornos. Apesar da conveniência de se utilizar esse modelo com fins descritivos, é preciso lembrar que suas fases nem sempre são distintamente separadas e podem variar muito de pessoa para pessoa ou, ainda, a cada encontro sexual. Tais variações, normais ou patológicas, devem sempre ser consideradas no diagnóstico de uma disfunção sexual.

Quanto ao número de homens que sofre de disfunções sexuais (DS), um estudo realizado em 2000 sobre o perfil sexual do brasileiro, que entrevistou 2.835 indivíduos ( $47 \%$ de homens e $53 \%$ de mulheres), apresentou os seguintes resultados sobre os transtornos sexuais disfunção erétil - 46,2\%; ejaculação precoce - 15,8\%; falta de desejo sexual - 12,3\% e disfunção orgásmica - 10\% (Abdo, Oliveira Jr, Scanavino, \& Martins, 2002). Para alguns autores a ejaculação precoce (EP) é a disfunção sexual mais comum entre homens do mundo inteiro (Carson \& Gunn, 2006, Hellstrom, 2009), mas sua prevalência talvez não esteja dimensionada corretamente, pois a falta de consenso para sua clara definição e classificação contribui para as discrepâncias estatísticas. (Cavalcanti, Serrano \& Lopes, 2005).

Os transtornos podem ser classificados de acordo com sua cronologia - primário ou secundário; sua frequência - generalizada ou situacional, e de acordo com sua etiologia - orgânica ou psicológica (DSM-IV, 1995). $\mathrm{Na}$ origem orgânica das DS podemos citar fatores como doenças cardíacas, vasculares, urológicas, proctológicas ou hormonais; diabetes, hipertensão ou insuficiência renal; alguns tipos de cânceres e seus tratamentos; cirurgias ou o uso de determinados medicamentos; o consumo crônico de álcool, tabaco e drogas ilícitas (Afif-Abdo, 2007; Berg, 2000).

Já os fatores psicogênicos podem incluir medo, culpa, falsas concepções e expectativas irreais quanto ao desempenho sexual; insatisfação com a parceira; abuso sexual; estresse, distorções sobre a imagem corporal, ansiedade e depressão (Kern, 2010). A investigação da etiologia de uma disfunção sexual deve ir além da saúde física, mesmo que a gênese seja exclusivamente orgânica. Os aspectos psíquicos devem ser sempre considerados e avaliados atenciosamente sempre que houver uma queixa sexual.

Segundo Cavalcanti \& Lopes (2005) não há enfermidade física que não traga comprometimento psíquico nem problemas psicológicos que não acarretem alguma reação física. A utilização do termo dificuldade sexual parece ser mais adequada por abarcar uma compreensão mais ampla sobre o impacto que a queixa sexual, pode ter na vida de uma pessoa. A problemática sexual, muitas vezes extrapola o aspecto físico da disfunção, originando problemas na vida profissional, nos relacionamentos familiares e sociais, na relação com seu/sua parceiro/a e em sua saúde psíquica (Feijó, 2007).

Segundo Fitonelli Jr. \& Capitão (2011), a função sexual foi reconhecida pela Organização Mundial da Saúde como um indicador de saúde e, consequentemente, a disfunção sexual passou a ser vista como um problema de saúde pública. É vasta a literatura sobre o impacto das doenças crônicas e seus tratamentos sobre a qualidade de vida (QV) e a função sexual de homens e mulheres. Podemos citar como exemplos estudos que investigaram a relação entre QV, satisfação sexual e doenças como depressão, insuficiência renal e câncer (Bertam \& Castro, 2010; Nóra, Zambone \& Facio Júnior, 2009; Rosen \& cols., 2004); pesquisas que avaliam o impacto de tratamentos na QV, tais como a prostatectomia radical (Coelho \& cols., 2003; Kamalov \& cols., 2005; Punnen, Cooperberg, Sadetsky \& Carroll, 2012; Van Andel \& cols, 2004); ou ainda, estudos que avaliam as alterações da função sexual inerentes ao envelhecimento (Sousa, 2008; Viana \& Madruga, 2008).

Contudo, um levantamento feito nas bases de dados BVS, LILACS e SCIELO, nos últimos dez anos, revelou a escassez de estudos que abordem a existência de um impacto direto, ou mesmo sobre a influência das disfunções sexuais na qualidade de vida dos homens. Em uma pesquisa realizada com 2.476 homens espanhóis, com idade variando entre 25 e 70 anos, seus autores concluíram que a disfunção erétil (DE) tem um impacto negativo sobre a $\mathrm{QV}$, tanto na dimensão mental quanto física, sendo mais evidente nessa última (Sánchez-Cruz \& cols., 2003).

Ribeiro \& Santos (2005) afirmam que as disfunções sexuais, em especial a DE, podem acarretar efeitos negativos sobre a autoimagem, perda de autoconfiança, ser fonte de estresse e depressão. Pode afetar a saúde em geral e prejudicar a qualidade de vida de seus portadores. Em sua pesquisa desenvolvida com 133 homens portugueses, com idade variando entre 21 e 78 anos, dos quais $32,8 \%$ apresentavam $\mathrm{DE}$, concluíram que esse grupo tinha valores mais baixos de $\mathrm{QV}$ do que os indivíduos sem disfunção, principalmente nas dimensões do componente físico. 
Em outra pesquisa realizada com 137 homens iranianos com queixas sexuais, seus autores sugerem que os efeitos adversos dos problemas sexuais podem ir além da depressão, podendo acarretar efeitos negativos sobre a autoimagem, perda de autoconfiança, ser fonte de estresse, afetando a saúde em geral. Concluíram que a qualidade de vida dos homens com DS é pior do que a de indivíduos sem problemas sexuais (Naeinian, Shaeiri \& Hosseini, 2011).

Em um estudo realizado com 106 homens americanos, dos quais 57 apresentavam disfunção erétil, constatou-se que os domínios emocionais avaliados pelo SF-36 foram mais afetados pela DE do que os domínios físicos. Seus autores afirmaram que os relacionamentos conjugais desses homens são interdependentes de sua função sexual, ou seja, podem ser prejudicados pela disfunção sexual (Litwin, Nied \& Dhanani, 1998).

Nenhuma pesquisa nacional sobre essa temática foi encontrada na base de dados acima citada. $\mathrm{O}$ presente estudo teve o objetivo de investigar o funcionamento sexual e a qualidade de vida de homens com dificuldades sexuais atendidos no serviço de sexualidade do CIESEX/UNIFESP.

\section{Método}

O projeto desta pesquisa foi aprovado pelo Comitê de Ética da UNIFESP, sob no 181521 . Trata-se de um estudo descritivo, utilizando-se análise descritiva, média, desvio padrão e porcentagem dos resultados obtidos.

\section{Procedimento}

Este trabalho foi desenvolvido no Centro de Intervenção e Estudos em Sexualidade Humana - CIESEX/UNIFESP, que oferece atendimento individual, familiar, conjugal e grupal gratuito a pessoas com dificuldades sexuais. Homens que procuraram essa instituição com queixa de disfunção sexual foram submetidos a uma triagem, composta por entrevista estruturada para a coleta de dados sociodemográficos e informações sobre a queixa apresentada.

Após a coleta dos dados, os entrevistados foram informados sobre o presente estudo e convidados a participarem do mesmo. Aceito o convite, o Termo de Consentimento Livre e Esclarecido foi apresentado para sua leitura e assinatura. Em seguida, foram entregues dois questionários para serem respondidos pelos próprios pacientes. Encerrado o preenchimento, os psicólogos esclareceram eventuais dúvidas e providenciaram o agendamento para posterior consulta.

\section{Participantes}

A amostra foi composta por dez pacientes que apresentavam queixas sexuais e obedeciam aos seguintes critérios de inclusão: idade superior a 40 anos, homens heterossexuais com parceria fixa, sem comorbidade psiquiátrica e disponibilidade para o atendimento no horário e dia oferecidos pela instituição.

Participaram deste estudo dez homens com idade variando entre 42 e 62 anos, com média de 52,8 anos (DP $\pm 13,32$ ). A partir dos dados coletados na entrevista, foi possível constatar que oito dos entrevistados eram casados. Entretanto, todos tinham relacionamento estável com parceira fixa, variando entre 6 a 36 anos, com média de anos 22,1 anos (DP $\pm 10,79)$. Quanto à religiosidade, quatro responderam ser católicos, dos quais três afirmaram não ser praticantes; quatro eram evangélicos, um respondeu ser espírita e um relatou não seguir nenhuma religião. Quanto à região de origem, sete são provenientes da região sudeste e três do nordeste.

Em relação ao grau de escolaridade dois dos entrevistados cursaram o ensino superior, quatro o ensino médio e quatro o ensino fundamental. Quanto à atividade profissional, cinco trabalhavam na área de transportes, dois na área comercial e os demais se dividiam nas áreas de administração, educação e industrial.

Quanto ao histórico de saúde, três homens afirmaram ter pressão alta e utilizarem captopril como tratamento, outros três relataram ter colesterol alto, seguindo uma dieta controlada. Todos negaram diabetes. Outras doenças relatadas foram: artrose $(n=2)$, gastrite $(n=2)$, ataxia $(n=1)$ e câncer de pele $(n=1)$. Três pacientes afirmaram ter contraído gonorreia na juventude. Todos negaram distúrbios miccionais ou doenças urológicas. Nove pacientes haviam realizado exame de próstata, sem qualquer alteração patológica.

Sobre o uso de drogas, seis pacientes não consumiam álcool e quatro afirmaram beber socialmente. Todos negaram tabagismo e apenas dois haviam fumado anteriormente. Dois pacientes relataram uso de drogas ilícitas anteriormente (maconha, cocaína e crack), mas no momento da entrevista nenhum deles fazia uso das mesmas.

\section{Instrumentos}

A ficha de triagem, preenchida pelos psicólogos da instituição, é composta por campos que abrangem informações sociodemográficas; histórico médico 
(doenças existentes, utilização de medicamentos e/ou drogas, tratamentos passados ou atuais, cirurgias, hábitos alimentares e qualidade do sono); histórico sexual (parceria, tempo de relação, frequência de relações sexuais, ciclo da resposta sexual, identificação e classificação da queixa e sua interferência na atividade sexual, durante o encontro sexual ou na masturbação).

Para investigar o funcionamento sexual, foi utilizado o instrumento The Golombok-Rust Inventory of Sexual Satisfaction (GRISS), um questionário psicometricamente construído e estandardizado, que possibilita a avaliação tanto do comportamento sexual quanto do nível da qualidade do funcionamento sexual. Sua tradução e adaptação para o português foi realizada em uma pesquisa com 626 sujeitos do Estado do Rio Grande do Sul (339 mulheres e 287 homens), com idades variando entre 18 e 74 anos (Diehl, 2000).

Composto por 28 itens para avaliar a existência e severidade de problemas sexuais, apresenta uma versão feminina e uma masculina. Ambas as versões têm sete subescalas, dais quais, cinco avaliam os mesmos índices de insatisfação sexual, não comunicação sexual, infrequência das relações sexuais, evitação sexual e falta de expressão da sensualidade. As disfunções sexuais são avaliadas separadamente para os homens incluem-se os itens de impotência e ejaculação precoce; já para as mulheres, são compreendidos os indicadores de anorgasmia e vaginismo (Diehl, 2000).

O GRISS é um instrumento de autopreenchimento, e a cada questão corresponde uma única alternativa (nunca, quase nunca, às vezes, frequentemente, sempre), com valores variando de 0 a 4 pontos. Segundo Diehl (2000), as respostas marcadas nos questionários são transformadas em seus valores correspondentes pela Tabela de Escores. Tais resultados são transformados em mais duas tabelas (Escores das Subescalas e de Transformações), chegando ao escore que indica o nível do funcionamento sexual de homens e mulheres.

A somatória dos pontos atingidos em cada subescala também é transformada em um único valor, denominado escore global, com variação entre 1 e 9 . Os valores a partir de cinco indicam a ocorrência de problemas sexuais. Quanto mais elevada for a pontuação atingida, menor é a qualidade do funcionamento sexual e/ou maior é o problema ou a dificuldade sexual.

O questionário Short Form Health Survey 36-item (SF-36) foi traduzido e adaptado para a população brasileira em um estudo realizado com 50 pacientes com artrite reumatoide, no qual seus autores concluíram ser ele um instrumento válido para a utilização no Brasil (Ciconelli, Ferraz, Santos, Meinão, \& Quaresma, 1999). O SF-36 pode ser aplicado pelo entrevistador ou respondido pelo entrevistado, não requerendo muito tempo para sua realização.

Composto por 36 questões e oito domínios que avaliam: capacidade funcional (dez itens que avaliam a capacidade ou dificuldade para executar atividades cotidianas); aspectos físicos (composto por quatro itens que identificam problemas em função do estado de saúde em atividades laborais ou cotidianas); dor (dois itens para identificar a existência de dor, sua intensidade e influência nas atividades diárias); estado geral de saúde (cinco itens que investigam a percepção e expectativas que a pessoa tem sobre sua saúde); vitalidade (quatro itens que refletem a percepção do indivíduo de sua energia e disposição para realizar atividades cotidianas), abarcando as condições físicas de saúde.

Já as condições mentais são avaliadas por meio dos seguintes tópicos: aspectos sociais (dois itens que investigaram o quanto problemas emocionais e saúde física interferem nos relacionamentos sociais e familiares); aspectos emocionais (três itens que refletem a interferência de problemas emocionais no cotidiano) e saúde mental (cinco itens que avaliam a percepção do indivíduo de seu humor e estado emocional). Cada domínio possui de 2 a 10 itens, sendo seus resultados expressos em uma pontuação que varia de 0-100, indo do pior para o melhor estado de saúde. Escores abaixo de 50 na escala indicam uma qualidade de vida abaixo da média (Caponilna \& Ciconelli, 2008; Soares, Toledo, Santos, Lima, \& Galdeano, 2008).

\section{Resultados}

A Tabela 1 apresenta dados sobre as fases da resposta sexual, na qual se observa que quatro pacientes responderam que sempre sentiam desejo, e destes, metade revelou que o mesmo diminuiu em virtude do quadro disfuncional.

Quanto à fase de excitação sexual, quatro dos entrevistados afirmaram se excitar sempre, e seis pacientes confirmaram ter ereção suficiente para penetração durante a relação sexual. Quando questionados sobre que nota poderia ser dada para a rigidez peniana, em uma escala de 0 a 10 (sendo 0 total flacidez e 10 total rigidez), as notas variaram nos dois extremos, sendo a média de 5,5. Cinco pacientes relataram que não conseguiam manter a ereção durante a relação sexual, quatro responderam que mantinham e um que às vezes 
conseguia outras não. A ereção matinal foi confirmada por sete pacientes e quatro afirmaram a ocorrência de ereções noturnas.

As queixas relatadas pelos pacientes durante a triagem foram: disfunção erétil $(n=3)$, ejaculação precoce $(n=6)$ e falta de desejo sexual $(n=1)$. A disfunção estava presente em todas as relações sexuais $(n=8)$. Nove relataram que o problema ocorreu após certo tempo de vida sexual satisfatória, caracterizando a queixa como secundária, com tempo de manifestação variando de 5 a 10 anos, sendo a média de 4,1 anos. Sete pacientes haviam procurado tratamento anterior: urologistas ou clínicas $(n=7)$, utilizando medicamento oral $(n=3)$ ou injeção $(\mathrm{n}=2)$; um paciente fez psicoterapia e um utilizou a acupuntura.
O uso do questionário GRISS possibilitou confirmar a ocorrência e avaliar a severidade dos problemas sexuais, revelando uma ampliação no número de queixas. A maioria dos pacientes $(n=6)$ pontuou tanto para DE quanto para EP, modificando assim o percentual das queixas desse grupo, como demonstrado na Tabela 2.

Os escores obtidos no GRISS confirmaram a queixa relatada por oito dos pacientes. Os que verbalizaram EP $(n=5)$ tiveram escore igual ou superior a sete pontos, sendo que três destes atingiram a pontuação máxima, revelando alta gravidade da disfunção ejaculatória. Os três pacientes que apresentaram DE pontuaram entre seis e sete pontos, indicando menor severidade da disfunção.

Tabela 1. Fases da resposta sexual

\begin{tabular}{lccccc}
\hline Resposta Sexual & $\mathrm{S}$ & $\mathrm{F}$ & $\mathrm{O}$ & $\mathrm{R}$ & $\mathrm{N}$ \\
\hline Desejo sexual & $4(40 \%)$ & $1(10 \%)$ & $2(20 \%)$ & $2(20 \%)$ & 0 \\
Excitação & $4(40 \%)$ & $1(10 \%)$ & 0 & $4(40 \%)$ & 0 \\
Orgasmo & $2(20 \%)$ & $1(10 \%)$ & 0 & $5(50 \%)$ & $1(10 \%)$ \\
\hline
\end{tabular}

Obs.: $\mathrm{S}=$ sempre, $\mathrm{F}=$ frequentemente $\mathrm{O}=$ ocasionalmente, $\mathrm{R}=$ raramente, $\mathrm{N}=$ nunca

Dois pacientes não tiveram sua queixa confirmada pelos resultados obtidos através do questionário GRISS. Um trouxe como queixa inicial a falta de desejo sexual, mas pontuou com problemas de ereção e descontrole ejaculatório e outro apresentou escore de apenas um ponto para EP (queixa verbalizada), mas de oito pontos para DE.

A Tabela 3 traz dados sobre a qualidade do funcionamento sexual desses homens. No item Falta de expressão de sensualidade masculina (FESM), o maior escore atingido pela maioria dos pacientes $(n=7)$ foi de sete pontos, revelando terem dificuldade para o envolvimento erótico com a companheira, como por exemplo, gostar de tocar os genitais da parceira, que ela toque seus genitais; gostar de abraçar ou acariciar, ser abraçado ou acariciado.

Metade dos pacientes $(n=5)$ pontuou no aspecto Evitação sexual masculina (EMS), que se refere a fatores como a presença de tensão ou ansiedade quando a parceira quer ter relações sexuais; tentativa de evitar ou

Tabela 2. Queixa verbalizada x queixa avaliada pelo questionário GRISS

\begin{tabular}{lcccc}
\hline Queixa & Verbalizada & $\%$ & Avaliada & $\%$ \\
\hline Disfunção erétil (DE) & 3 & 30 & 2 & 20 \\
Ejaculação precoce(EP) & 6 & 60 & 2 & 20 \\
Falta de desejo sexual & 1 & 10 & 0 & 0 \\
DE + EP & 0 & 0 & 6 & 60 \\
Total & 10 & 100 & 10 & 100 \\
\hline
\end{tabular}


recusa de ter relação sexual; sensação de desconforto com o que é feito durante o coito. E na avaliação da Insatisfação sexual masculina (ISM), na qual fatores como variação do jogo sexual, satisfação com a frequência sexual, presença ou ausência de amor e afeição no relacionamento sexual, cinco pacientes atingiram escore superior a cinco pontos.

No item Infrequência de Relações sexuais (IRS), a maioria dos pacientes $(n=7)$ obteve escore acima de cinco pontos, indicando a ausência de relações sexuais em determinadas semanas. Três dos pacientes tiveram pontuação máxima, revelando, assim, a diminuição acentuada dos encontros sexuais. Na entrevista, todos os pacientes afirmaram que a frequência das relações sexuais era semanal, realidade esta que não foi confirmada pela aplicação do questionário, porém todos assinalaram uma diminuição da mesma decorrente da disfunção sexual.

A Não comunicação sexual (NCO) refere-se tanto à dificuldade de dizer à parceira quanto de perguntar a ela sobre o que gosta ou não na relação sexual. Dos seis pacientes que atingiram pontos iguais ou superiores a cinco, dois tiveram pontuação máxima, revelando que nunca conversaram sobre esse tema com a companheira.

Tabela 3. The Golombok-Rust Inventory of Sexual Satisfaction (GRISS)

\begin{tabular}{|c|c|c|c|c|}
\hline \multirow{2}{*}{ Domínio } & \multicolumn{2}{|c|}{$<5$} & \multicolumn{2}{|c|}{$\geq 5$} \\
\hline & $\mathrm{N}$ & $\%$ & $\mathrm{~N}$ & $\%$ \\
\hline Falta de expressão de sensualidade masculina (FESM) & 1 & 10 & 9 & 90 \\
\hline Evitação sexual masculina (ESM) & 5 & 50 & 5 & 50 \\
\hline Insatisfação sexual masculina (ISM) & 5 & 50 & 5 & 50 \\
\hline Infrequência de relações sexuais (IRS) & 3 & 30 & 7 & 70 \\
\hline Não comunicação sexual (NCO) & 4 & 40 & 6 & 60 \\
\hline
\end{tabular}

O SF-36 foi utilizado com o objetivo de avaliar a qualidade de vida dos homens com queixas sexuais e os resultados obtidos com sua aplicação são apresentados na Tabela 4. Tendo como referência a pontuação de 0-100 pontos, sendo zero a pior e cem a melhor condição de saúde, 50 pontos são utilizados como limite indicativo de um estado abaixo da média. Quatro pacientes não apresentaram escores que indicassem baixa qualidade de vida.

Dos seis pacientes que pontuaram abaixo de 50 , no que se refere ao componente físico, que avalia o impacto da saúde física, um paciente pontuou na dimensão dor,

Tabela 4. Short Form Health Survey 36-item (SF-36)

\begin{tabular}{lcccc}
\hline Domínios & Média & Mínimo & Máximo & DP \\
\hline Capacidade funcional & 78,0 & 30,0 & 100,0 & 25,7 \\
Limitação (asp. físicos) & 65,0 & 0,0 & 100,0 & 41,2 \\
Dor & 69,4 & 31,0 & 100,0 & 21,8 \\
Estado geral de saúde & 72,1 & 35,0 & 97,0 & 22,1 \\
Vitalidade & 66,5 & 30,0 & 100,0 & 21,1 \\
Aspectos sociais & 75,0 & 37,5 & 100,0 & 22,0 \\
Aspectos emocionais & 75,0 & 37,5 & 100,0 & 22,0 \\
Saúde mental & 60,4 & 28,0 & 100,0 & 24,6 \\
\hline
\end{tabular}


dois em estado geral de saúde, dois na dimensão capacidade funcional e três nos aspectos físicos. Dois dos pacientes que obtiveram pontuação elevada apresentavam doenças físicas que comprometiam a capacidade de locomoção e equilíbrio, o que pode ter influenciado os resultados.

Os pacientes tiveram pontuação abaixo de 50 nos seguintes domínios: um paciente em aspectos sociais, dois pacientes referiram ter pouca energia e disposição para atividades cotidianas, três pacientes assinalaram a influência dos problemas emocionais na rotina profissional e/ou doméstica. Quanto ao domínio saúde mental que reflete a percepção que o indivíduo tem em relação ao seu humor e ao seu estado emocional tivemos o maior número $(\mathrm{n}=5)$ de pacientes com baixa pontuação e, consequentemente, a menor média de todas as dimensões avaliadas pelo SF-36.

\section{Discussão}

A existência de doenças orgânicas, o uso de medicamentos, de drogas lícitas ou ilícitas, são aspectos que devem ser investigados na vida do homem que apresenta dificuldades sexuais, pois pode prejudicar o processo de excitação, levando à disfunção. Nesta amostra, nenhum dos pacientes apresentou um quadro de saúde física com elementos que pudessem ser interpretados como causa(s) da problemática manifesta.

A investigação sobre as fases da resposta sexual revelou que, na percepção dos homens entrevistados, todas sofreram algum comprometimento por causa da disfunção. A maioria afirmou que o desejo diminuiu, bem como a excitação, pois aumentou a dificuldade para experimentarem uma ereção totalmente rígida. Também relataram ter, em algumas relações, reduzida a capacidade de manter a ereção, perdendo-a antes ou durante a penetração.

Ao não ter uma excitação adequada e, consequentemente, não conseguir atingir uma rigidez plena, o homem pode ter dificuldade para chegar ao clímax e à experiência do prazer intenso. Nesse grupo, a vivência do orgasmo foi prejudicada, sendo que metade dos pacientes relatou que raramente sentiam prazer. Tal dado revela que alterações na capacidade funcional têm impacto sobre a percepção que o homem tem do prazer vivido durante o encontro sexual.

Não raro, a ejaculação precoce aparece como uma disfunção secundária à incapacidade de uma excitação adequada. Carson e Gunn (2006) afirmam que DE e EP coexistem frequentemente, sendo inegável que a existência da disfunção erétil leva ao risco do desenvolvimento da ejaculação precoce. Isso pode ocorrer, pois, ao buscarem uma estimulação mais intensa, em uma tentativa de não perder a ereção durante a penetração, aceleram a resposta ejaculatória (Althof \& cols., 2010). Nessa amostra, a maioria dos entrevistados apresentou EP como queixa, contudo, com a aplicação do questionário GRISS, obteve-se uma alteração nesse quadro, identificando-se que a maioria apresentava ambas as disfunções.

Repetidas experiências negativas durante o encontro sexual, frustrações, decepções, cobranças explícitas ou veladas podem gerar temores e receios sobre as próximas relações sexuais. Compõe-se, assim, um quadro que pode levar à diminuição acentuada do desejo sexual (Lucas, Oliveira \& Monteiro, 2009) e, consequentemente, gerar insatisfação com o relacionamento sexual.

Segundo Diehl (2000), quanto mais altos são os valores obtidos no questionário GRISS, menor é a qualidade do funcionamento sexual. $\mathrm{O}$ domínio mais prejudicado nessa amostra refere-se à expressão de sensualidade masculina, indicando a dificuldade do homem em se envolver intimamente com a parceira no que se refere ao jogo erótico, à exploração de diferentes formas de estimulação e de outras fontes de prazer, além do intercurso sexual. Soma-se a esse quadro tanto a dificuldade de dizer à parceira quanto de perguntar a ela sobre o que gosta ou não na relação sexual, também assinalado como um dos domínios de maior pontuação em nossa amostra.

Em razão da preocupação com o desempenho sexual, o medo do fracasso, o receio de novas frustrações, homens com DS sofrem prejuízos em sua autoestima e em sua autoconfiança, (Martín-Morales, Rico, González, Anechina \& Font, 2005). Tal quadro pode desencadear vergonha e ansiedade, induzindo à insatisfação com a relação sexual e à falta de intimidade com a parceira, podendo culminar com o fim do relacionamento (Revicki \& cols., 2008). Não apenas há um prejuízo na qualidade, mas também uma diminuição no número de intercursos, ocasionando a evitação do encontro sexual e uma redução em sua frequência, realidade esta trazida pelos pacientes durante a entrevista e confirmada com a aplicação do GRISS.

Como os pacientes, em sua grande maioria, pontuaram acima de cinco em todos os aspectos avaliados, constata-se que essa amostra apresentou uma qualidade do funcionamento sexual bastante prejudicada. A dificuldade para o envolvimento erótico com a parceira, problemas de comunicação e insatisfação com 
a frequência sexual trazem à tona a necessidade de se avaliar se a deterioração da comunicação, da intimidade entre o casal pode contribuir para o agravamento da dificuldade sexual. Os estudiosos Litwin, Nied \& Dhanani (1998) afirmam que a problemática sexual influencia negativamente o relacionamento afetivo-sexual dos casais.

Segundo Scorsolini-Comin e Santos (2010) o relacionamento conjugal está associado à qualidade de vida, principalmente nos anos de maturidade e velhice, sendo fundamental para o bem-estar psicológico e social das pessoas. Segundo Finotelli Jr \& Capitão (2011), a avaliação das DS deve ter como principal foco o funcionamento sexual, seu desempenho e a satisfação do homem com o relacionamento afetivo-sexual, pois tal quadro não compromete apenas sua satisfação, mas também se associa à baixa autoestima e baixa qualidade de vida.

Os dados sobre a QV desta amostra revelaram que o domínio saúde mental, que reflete a percepção que o indivíduo tem em relação ao seu humor e ao seu estado emocional, teve a menor média de todas as dimensões avaliadas. Os estudos realizados sobre o impacto das DS sobre a qualidade de vida também referem prejuízo nas dimensões avaliadas, contudo, ressaltando que o componente físico é o mais afetado, principalmente pela disfunção erétil (Naeinian, Shaeiri \& Hosseini, 2011; Ribeiro \& Santos, 2005; Sánchez-Cruz \& cols., 2003).

Este estudo exploratório revelou aspectos importantes sobre o funcionamento sexual, o relacionamento afetivo-conjugal e a QV de homens com dificuldades sexuais. Por se tratar de uma amostra com um pequeno número de pacientes, os resultados obtidos devem ser analisados com cautela e sem generalizações.

Observou-se uma baixa qualidade do funcionamento sexual e uma menor média dos aspectos emocionais na investigação da QV, contudo não foram feitas análises correlacionando o funcionamento sexual com a qualidade de vida. Tais constatações indicam a necessidade de estudos mais abrangentes, tanto no que se refere ao número de sujeitos quanto na relação entre os aspectos investigados, explorando o impacto das dificuldades sexuais nas mais variadas dimensões na vida do homem. O desenvolvimento de futuras pesquisas pode contribuir para a construção de bases de conhecimento que ampliem a atuação de profissionais na área da sexualidade humana.

\section{Referências}

Abdo, C. H. N., Oliveira Jr., W. M., Moreira, E. D., \& Fittipaldi, J. A. S. (2002). Perfil sexual da população brasileira: resultados do estudo do comportamento sexual (ECOS) do brasileiro. Rev Bras Med., 59(4), 250-257.

Afif-Abdo, J. (2007). Diagnóstico e tratamento da disfunção erétil. Revista Diagnóstico Tratamento, 12(4), 192-195.

Althof, S. E., Abdo, C. H. N., Dean, J., Hackett, G., McCabe, M.,....\& McMahon, C. G. (2010). International society for sexual medicine's guidelines for the diagnosis and treatment of premature ejaculation. J. Sex. Med., 7, 2947-2969.

American Psychiatric Association (1995). DSM-IV. Manual diagnóstico e estatístico de transtornos mentais. Trad. Dayse Batista. ( $4^{\mathrm{a}}$ ed.). Porto Alegre: Artes Médicas.

Berg, O. (2000). Afinal, o que é disfunção erétil? Verdades e mentiras sobre impotência sexual. Rio de Janeiro: Dunya.

Bertan, F. C., \& Castro, E. K. (2010) Qualidade de vida, indicadores de ansiedade e depressão e satisfação sexual em pacientes adultos com câncer. Revista $S a$ lud \& Sociedad, 1(2), 76-88.

Britto, R., \& Benetti, S. P. C. (2010). Ansiedade, depressão e característica de personalidade em homens com disfunção sexual. Rev. SBPH, 13(2), 243-285.

Caponilna, A. G., \& Ciconelli, R. M. (2008). O SF-36 e o desenvolvimento de novas medidas de avaliação de qualidade de vida. Acta Reumatológica Portuguesa, 33, 127-133.

Carson C., \& Gunn, K. (2006). Premature ejaculation: definition and prevalence. Int J Impot Res., 18(Suppl 1), S5-13.

Cavalcanti, R. C., \& Lopes, G. P. (2005). Tratamento psicológico da disfunção erétil: manual prático de orientação clínica. São Paulo: Ponto.

Cavalcanti, R. C., Serrano, R. H., \& Lopes, G. P. (Eds.). (2005). Ejaculação precoce/rápida. Rio de Janeiro: Guanabara Koogan.

Ciconelli, R. M., Ferraz, M. B., Santos, W., Meinão, I., \& Quaresma, M. R. (1999). Tradução para a língua portuguesa e validação do questionário genérico de avaliação 
de qualidade de vida SF-36 (Brasil SF-36). Rev. Bras. Reumatol., 39(3), 143-50.

Coelho, J. C. U., Matia, J. E. F., Zeni Neto, C., Godoy, J. L., Canan Junior, L. W., \& Jorge, F. M. F. (2003). Função sexual de homens submetidos a transplante hepático. Rev. Assoc. Med. Bras. 49(4), 413-417.

Diehl, J. A. (2000). A qualidade do funcionamento sexual de homens e mulheres. Dissertação de Mestrado. Pontifícia Universidade Católica do Rio Grande do Sul, Porto Alegre, RS, Brasil.

Feijó, M. R. (2007). Práticas sistêmicas com casais e famílias com dificuldades afetivo-sexuais. Em A. L. M. Horta, \& M. R. Feijó (Orgs.), Sexualidade na família. (pp. 111-124). São Paulo: Expressão e Arte.

Finotelli Jr., I., \& Capitão, C. G. (2011). Evidências de validade da versão brasileira da Escala de Autoeficácia Sexual -

Função Erétil. Psico-USF, 16(1), 45-55.

Gherpelli, M. H. B. V. (1995). Diferente, mas não desigual: a sexualidade no deficiente mental ( $2^{\mathrm{a}}$ ed.). São Paulo: Gente.

Hellstrom, W. J. G. (2009). Emerging treatments for premature ejaculation: focus on dapoxetine. Neuropsychiatr. Dis. Treat. 5, 37-46.

Kamalov A. A., e cols. (2005). Quality of life in patients after radical prostatectomy. Urologiia. 4, 27-9.

Kaplan, H. S. (1977). A nova terapia do sexo. (O. B. Silva, Trad.). Rio de Janeiro: Nova Fronteira. (Original publicado em 1974).

Kern, C. A. R. (2010). Disfunção Sexual Masculina: Compreensão psicanalítica. Dissertação de Mestrado. Universidade do Vale do Rio dos Sinos, São Leopoldo, RS, Brasil.

Litwin, M., Nied, R. J., \& Dhanani, N. (1998). HealthRelated quality of life in men with erectile dysfunction. Journal of General Internal Medicine, 13(3), 159-166.

Lucas, C. O., Oliveira, C. M. \& Monteiro, M. I. A. (2009). Perturbação do desejo sexual hipoativo: prevalência, diagnóstico e tratamento. Mudança-Psicologia da Saúde, 17(2), 101-112.

Martín-Morales, A., Rico, F. M., González, J. I. G., Anechina, L. R, \& Font, M. M. (2005). Repercusiones psicológicas de la disfunción eréctil sobre la autoestima y autoconfianza. Actas Urol. Esp. 29(5), 493-498.

Masters, W. H., \& Johnson, V. E. (1984). A resposta sexual humana. (A. A. T. Serra, Trad.) São Paulo: Roca. (Original publicado em 1966).

Naeinian, M. R., Shaeiri, M. R., \& Hosseini, F. S. (2011). General health and quality of life in patients with sexual dysfunctions. Urol. J. 8, 127-131.

Nóra, R. T., Zambone, G. S., \& Facio Júnior, F. N. (2009). Evaluation of quality of life and sexual dysfunctions in chronic renal failure patients undergoing hemodialysis in a hospital. Arq. Ciênc. Saude. 16(2), 72-75.

Punnen S., Cooperberg M. R., Sadetsky N., \& Carroll P. R. (2012). Among potent men post radical prostatectomy, does the need for phosphodiesterase inhibitors have an impact on sexual bother scores? BJU Int., 109(10), 1520-1524.

Revicki D., Howard K., Hanlon J., Mannix S., Greene A., \& Rothman M. (2008). Characterizing the burden of premature ejaculation from a patient and partner perspective: a multi-country qualitative analysis. Health and Quality Life Outcomes. 6, 33.

Ribeiro, J. P., \& Santos, A. (2005). Estudo exploratório da relação entre função eréctil, disfunção eréctil e qualidade de vida em homens portugueses saudáveis. Análise Psicológica, 3(23), 341-349.

Rosen, R. C., e cols. (2004). Quality of life, mood, and sexual function: a path analytic model of treatment effects in men with erectile dysfunction and depressive symptoms. Int.J.Impot.Res. 1, 334-340.

Sánchez-Cruz J. J., Cabrera-León, A., Martín-Morals, A., Fernández, A., Burgos, R., \& Rejas, J. (2003). Male erectile dysfunction and health-related quality of life. Eur. Urol., 44(2), 245-53.

Scorsolini-Comin, F., \& Santos, M. A. (2010). Satisfação conjugal: revisão integrativa da literatura científica nacional. Psicologia: Teoria e Pesquisa, 26(3), 525-531.

Seidl, E. M. F, \& Zannon, C. M. L. C. (2004). Qualidade de vida e saúde: aspectos conceituais e metodológicos. Cad. Saúde Pública. 20(2), 580-588.

Soares, D. A., Toledo, J. A. S., Santos, L. F., Lima, R. M. B., \& Galdeano, L. E. (2008). Qualidade de vida 
de portadores de insuficiência cardíaca. Acta Paul Enferm., 21(2), 243-248.

Sousa J. L. (2008). Sexualidade na terceira idade: uma discussão da AIDS, envelhecimento e medicamentos para disfunção erétil. J Bras Doenças Sex Transm., 20(1), 59-64.

Van Andel G. e cols. (2004). A prospective longitudinal study comparing the impact of external radiation therapy with radical prostatectomy on health related quality of life (HRQOL) in prostate cancer patients. Prostate, 58(4), 354-365.

Viana, H. B., \& Madruga, V. A. (2008). Sexualidade, qualidade de vida e atividade física no envelhecimento. Conexões: Revista da Faculdade de Educação Física da UNICAMP, 6, 222-233.

Recebido em: 22/03/2013

Reformulado em: 16/10/2013

Segunda reformulação em: 10/12/2013

Aprovado em: 19/02/2014

Sobre os autores:

Maria Cristina Romualdo Galati é psicóloga, psicodramatista, mestre em Ciências pela UNIFESP e colaboradora do Instituto Kaplan - Centro de Estudos da Sexualidade Humana e do CIESEX /UNIFESP. Cocriadora dos materiais educativos Jogo de Corpo e Aprendendo a Viver, do Instituto Kaplan. É psicoterapeuta e terapeuta sexual em consultório particular.

Enaury de Oliveira Alves Junior é psicólogo colaborador da disciplina de Urologia (Uro-Onco e Disfunção Erétil) do Hospital São Paulo e no Centro de Intervenção e Estudos em Sexualidade Humana - CIESEX /UNIFESP e pós-graduando nível Doutorado na UNIFESP/EPM.

Ana Claudia Crotti Delmaschio é psicóloga clínica, especialista em Sexualidade Humana/FMUSP.

Ana Lucia de Moraes Horta é doutora em enfermagem, terapeuta familiar e de casal, psicidramatista, professora adjunta da Escola Paulista de Enfermagem EPE/ UNIFESP, coordenadora do curso de Especialização em terapia familiar e de casal da UNIFESP.

\section{Contato com os autores:}

Centro de Intervenção e Estudos de Sexualidade Humana (CIESEX)

Escola de Enfermagem - Universidade Federal de São Paulo.

Rua - Leandro Dupret, 166 - Vila Clementino - São Paulo - SP - CEP: 04025-010.

E-mail: analuciahorta18@gmail.com 